\title{
Polar Vortexes and Charged Domain Walls in a Room Temperature Magnetoelectric Thin Film
}

Kalani Moore ${ }^{1 *}$, Eoghan N. O'Connell ${ }^{1}$, Lynette Keeney ${ }^{2}$, Clive Downing ${ }^{3}$, Michael Schmidt ${ }^{2}$, Valeria Nicolosi, ${ }^{3,4}$ Ursel Bangert $^{1}$, Michele Conroy ${ }^{1 *}$

1. Department of Physics, Bernal Institute, School of Natural Sciences, University of Limerick, Ireland 2. Tyndall National Institute, University College Cork, Ireland

3. Advanced Microscopy Laboratory \& AMBER, Trinity College Dublin, Dublin 2, Ireland

4. School of Chemistry, Trinity College Dublin, Dublin 2, Ireland

\begin{abstract}
Multiferroic domain walls are an emerging solution for future low-power nanoelectronics due to their combined tuneable functionality and mobility. Here we show that the magnetoelectric multiferroic Aurivillius phase $\mathrm{Bi}_{6} \mathrm{Ti}_{x} \mathrm{Fe}_{y} \mathrm{Mn}_{z} \mathrm{O}_{18}$ (B6TFMO) crystal is an ideal platform for domain wall-based nanoelectronic devices. The unit cell of B6TFMO is distinctive as it consists of a multiferroic layer between dielectric layers. We utilise atomic resolution scanning transmission electron microscopy and spectroscopy to map the sub-unit-cell polarisation in B6TFMO thin films. $180^{\circ}$ charged head-to-head and tail-to-tail domain walls are found to pass through $>8$ ferroelectric-dielectric layers of the film. They are structurally similar to $\mathrm{BiFeO}_{3} \mathrm{DWs}_{\text {b }}$ but contain a large surface charge density $\left(\sigma_{\mathrm{s}}\right)=1.09 \mid$ e| per perovskite cell, where $|\mathrm{e}|$ is elementary charge. Although polarisation is primarily in-plane, c-axis polarisation is identified at head-to-tail domain walls with an associated electromechanical coupling of strain and polarisation. Finally, we reveal that with controlled strain engineering during thin film growth, room-temperature vortexes are formed in the ferroelectric layer. These results confirm that sub-unit-cell topological features can play an important role in controlling the conduction properties and magnetisation state of Aurivillius phase films and other multiferroic heterostructures.
\end{abstract}




\section{Introduction}

Layered oxide thin films such as the $\mathrm{Bi}_{2} \mathrm{O}_{2}\left(\mathrm{~A}_{m-1} \mathrm{~B} \mathrm{O}_{3 m+1}\right)$ Aurivillius phase (AP) offer a flexible template for designing new multiferroic devices [1,2]. APs are a promising ferroelectric material with strong polarisation[3], and fatigue-free energy storage performance[4-6]. The rare possibility of room temperature magnetoelectric coupling is achieved in APs by simply substituting the structure with magnetic ions [7-11]. The room temperature magnetoelectric multiferroic property indicates that this material system has a very clear potential for future low energy devices such as a charge-to-spin conversion node in magnetoelectric-spin orbit logic for recurring neural networks $[12,13]$.

$\mathrm{Bi}_{6} \mathrm{Ti}_{2.8} \mathrm{Fe}_{1.52} \mathrm{Mn}_{0.68} \mathrm{O}_{18}$ (B6TFMO) is an example of an ion-substituted AP with experimentally proven reliable magnetoelectric switching [14,15]. B6TFMO can be thought of as a 2D nanostructured framework, with 5 PK-cells of ferroelectric (FE) $\mathrm{BiFeO}_{3}$ (BFO) sandwiched between dielectric (DE) $\mathrm{Bi}_{2} \mathrm{O}_{2}$ layers. $\mathrm{Mn}$ and $\mathrm{Ti}$ are substituted for Fe at some " $\mathrm{B}$ sites" and the preference for these magnetic cations to concentrate in the central PK-cell of the B6TFMO structure causes true room temperature ferromagnetism (FM), as opposed to the antiferromagnetic room temperature BFO [16]. The fundamental physics and growth conditions governing magnetoelectric properties are still not fully understood and thus methods to improve these results are difficult to plan. As the multiferroic-dielectric heterostructure is at the sub-unit cell scale $[17,18]$, probing any changes within this heterostructure requires the characterisation technique to be at atomic scale spatial resolution.

Initial aberration corrected scanning transmission electron microscopy (STEM) and spectroscopy revealed the preferential Mn cation segregation at the central PK layers across all samples [19]. In the regions where out-of-phase boundaries (OPBs) and associated stacking default defects were present, the magnetic $\mathrm{Mn}$ and Fe ion partitioning increased. It has been suggested that OPBs suppress the ferroelectricity [20], while their high structural disorder and elevated magnetic ion density increases the magnetic spin $[9,21]$. Thus, inducing higher OPB densities could result in improved magnetoelectric measurements and device efficiency. In this study, we focus on the role these OPB defects play in the formation of charged domain wall (DW) and polar vortexes. We map out the pico-meter scale atomic column shifts and unit 
cell deformation as demonstrated previously for other ferroic materials [22-26], revealing the direct link between OPBs and polar topological solitons in AP thin films.

\section{Results and Discussion}

The samples were grown by liquid injection chemical vapour deposition as described in reference [15]. Figure 1 shows a model of the five-layered B6TFMO unit cells alongside an atomic resolution scanning transmission electron microscopy (STEM) high-angle annular dark-field (HAADF) image of the structure. The FE-DE heterostructure within the B6TFMO unit cell leads to distinctive FE behaviour, where oppositely polarised PK-cells co-exist side by side within the FE-layer as demonstrated in Figure 1(b). The PK-cell is pseudo-cubic with a FE dipole forming analogous to that in $\mathrm{BiFeO}_{3}(\mathrm{BFO})$. The outer PK-cells bonded to the DE layer are hyper-tetragonal and thus highly strained along the c-axis $\left(\varepsilon_{y y}\right)$ compared to $\mathrm{BFO}, \mathrm{BiTiO}_{3}$ or $\mathrm{PbTiO}_{3}$ unit cells. At these outer PK-cells the polarisation is larger and always points towards the DE layer forming a "mirror plane". At the 3 PK-cells in the centre of the layer the $\varepsilon_{y y}$ strain relaxes and thus the polarisation state has a shallower potential well[27]. In other words, with less $\varepsilon_{y y}$ strain present, polarisation direction is largely determined by electrostatic stress and has a lower energy cost for switching. In line with the reduced dimension effects of thin lamellae seen in other ferroelectrics[28], the central and intermediate PK-cells prefer to have an in-plane polarisation, left or right as in Figure 1(b). Accordingly, AP has been observed to strongly favour in-plane polarisation macroscopically, measured as $50 \mu \mathrm{C} \mathrm{cm}{ }^{-2}$ in B6TFMO[14]. AP DWs have previously been viewed on a microscale and from the surface by piezoresponse force microscopy (PFM) but only FE switching across dielectric layers has been observed by STEM HAADF, where the dipole experiences an interruption rather than a continuous rotation across PK-cells[3,29]. The conventional approach to map out atomic resolution polarisation is by reversing the measured B-site displacement from STEM HAADF images. This is a reliable technique for AP materials and is used throughout this paper[29]. The large yellow arrows in Figure 1(a) and throughout this paper indicate the interpreted net polarisation vector for each FE layer. 
(a)

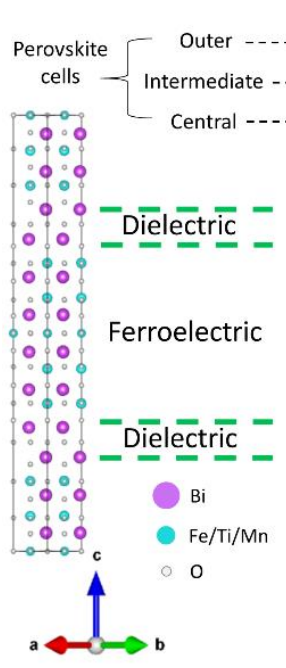

(b)

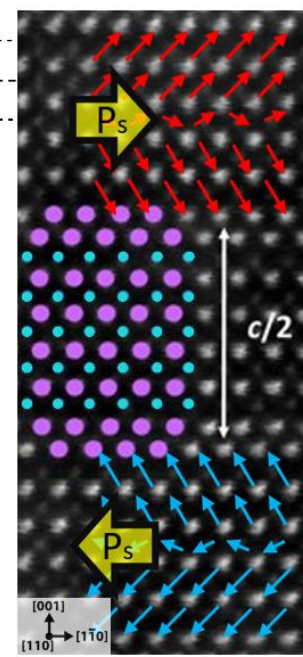

(c)

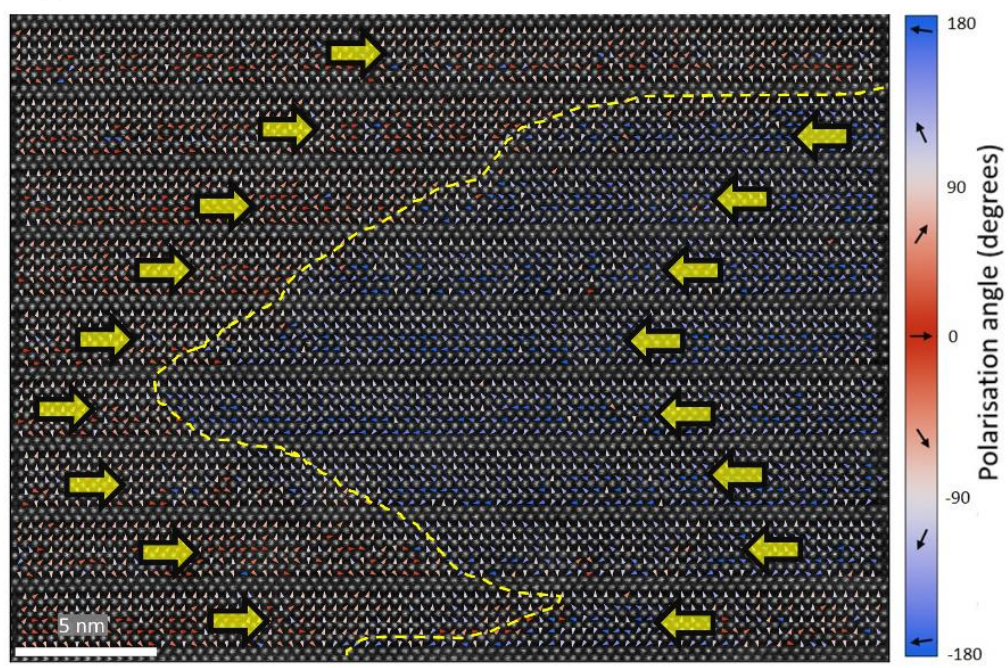

Figure 1. (a) Structural model of the five-layered Aurivillius phase B6TFMO unit cell (polar orthorhombic space group F2mm). (b) The A-site and B-site atoms in the structure are overlaid on an atomic resolution high angle annular dark field image. Red/blue arrows are an illustration of the expected polarisation of the perovskite (PK)-cells in the ferroelectric layer. The resulting in-plane net polarisation $\left(P_{s}\right)$ is indicated by the large yellow arrows. The ferroelectric and dielectric layers can be clearly distinguished by the Bi atom structures. (c) High angle annular dark field image overlaid with reverse B-site displacement polarisation map of each PK-cell. Polarisation unit vectors are colour coded red/blue to illustrate the in-plane polarisation direction. The approximate position of a headto-head $180^{\circ}$ domain wall is marked by the dashed yellow line. Scalebar $=5 \mathrm{~nm}$. See Supplementary Figures 1 \& 2 for maps of the polarisation averaged over 5 x 5 PK-cells in the ferroelectric layer.

Nominally charged DWs have been observed in other AP materials such as 4-layered $\mathrm{Bi}_{5} \mathrm{Ti}_{3} \mathrm{FeO}_{15}$ but only from the surface by PFM [3]. In Figure 1(c) we observe an atomic resolution vertical DW passing through multiple AP unit cells. Net polarisation here is headto-head $(\mathrm{H}-\mathrm{H})$ oriented and rotates $180^{\circ}$ across the DW. The DW is thus strongly charged[30]. The depth of this wall indicates that FE layers are coupled, not fully electrostatically isolated by the DE layers in between. The blue/red colouring of the polarisation unit vectors indicates the in-plane vector component to the left/right respectively. H-H DWs have "bound positive charge" from the adjacent polarisations which should be screened by a negative surface charge density $\left(\sigma_{\mathrm{s}}\right)$ equal to $2 \times \mathrm{P}_{\mathrm{s}}[30]$. 
In the case of B6TFMO where in-plane $P_{s}=0.5 \mathrm{C} / \mathrm{m}^{2}$,

$$
\sigma_{\mathrm{s}}=2 \times 0.5 \mathrm{C} / \mathrm{m}^{2}=1 \mathrm{C} / \mathrm{m}^{2}
$$

To make sense of what this means on the atomic scale we convert it to units of |e|/PK-cell. Where $|\mathrm{e}|=1.6 \times 10^{-19}$, or elementary charge, and PK-cell is the average surface area of a PKcell, approximately $3.835 \AA$ × $4.5 \AA$. Then,

$$
\sigma_{\mathrm{S}}=\frac{(1)\left(3.835 \times 10^{-10}\right)\left(4.5 \times 10^{-10}\right)}{\left(1.6 \times 10^{-19}\right)}=1.09|\mathrm{e}| / \text { PK-cell. }
$$

This calculated $\sigma_{\mathrm{S}}$ is of the same order as that found at strongly charged DWs in other perovskites[31]. As charge is likely to be spread across the DW width for 5-8 PK-cells, rather than confined to a 2D surface, a more realistic number for charge density $(\rho)$ would be

$$
\rho=\frac{1.09}{6.5 \text { PK cells }}=0.17|\mathrm{e}| / \text { PK-cell. }
$$

However, this value would vary depending on the local DW width. The screening charge could be provided by either $[\mathrm{Bi} / \mathrm{Ti} / \mathrm{Mn} / \mathrm{Fe}]$ vacancies or a local change in oxidation state $[\mathrm{Bi} / \mathrm{Ti} / \mathrm{Mn} / \mathrm{Fe}]^{3+} \rightarrow[\mathrm{Bi} / \mathrm{Ti} / \mathrm{Mn} / \mathrm{Fe}]^{4+}+1 e^{-}$. The existence of two-dimensional sheet of charge carriers passing through layers of a multiferroic film raises the possibility of electroresistance complementing magnetoresistance in a multiferroic tunnel junction memory device[32][33]. Measuring the electric field influence on the magnetisation through magnetoelectric coupling and the exact screening charge at the DW will be the subject of future research. 
(a)

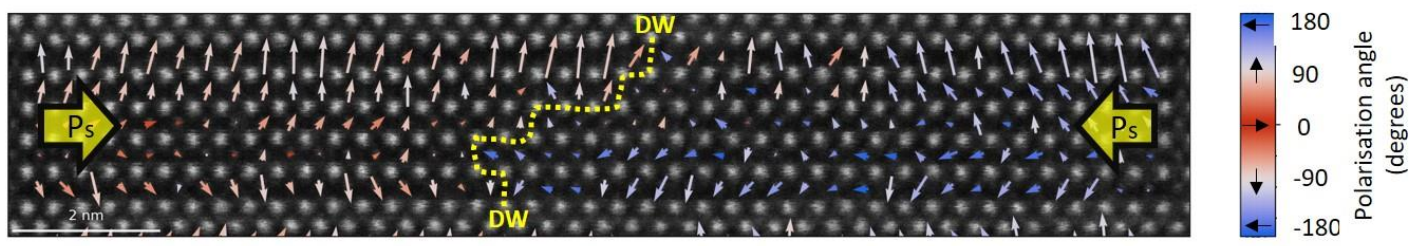

(b)

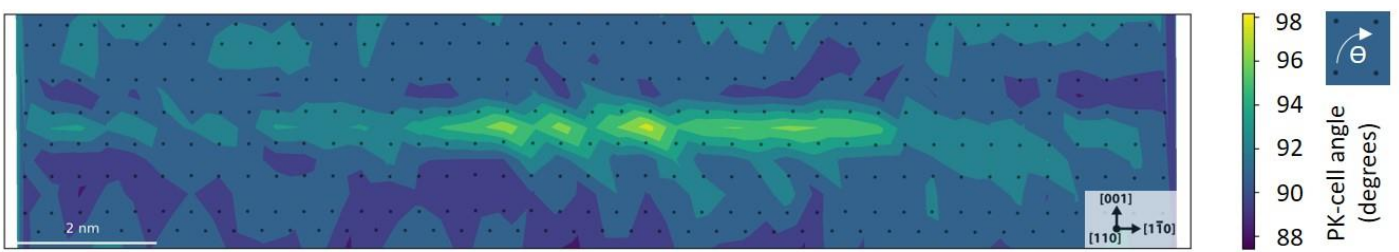

(c)

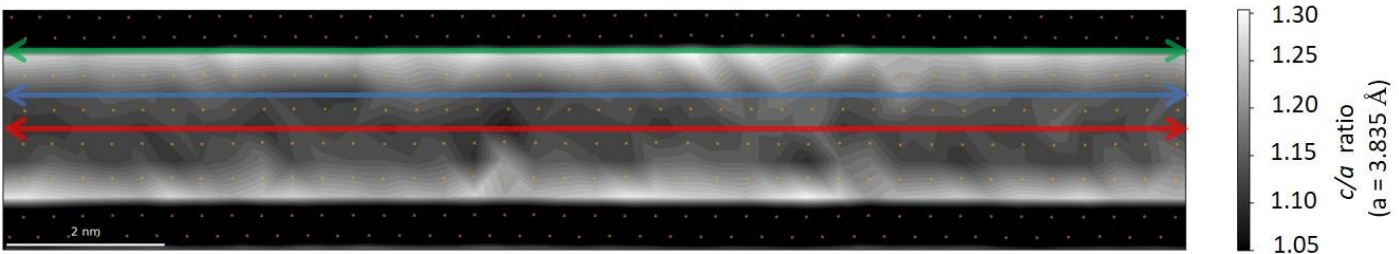

(d)

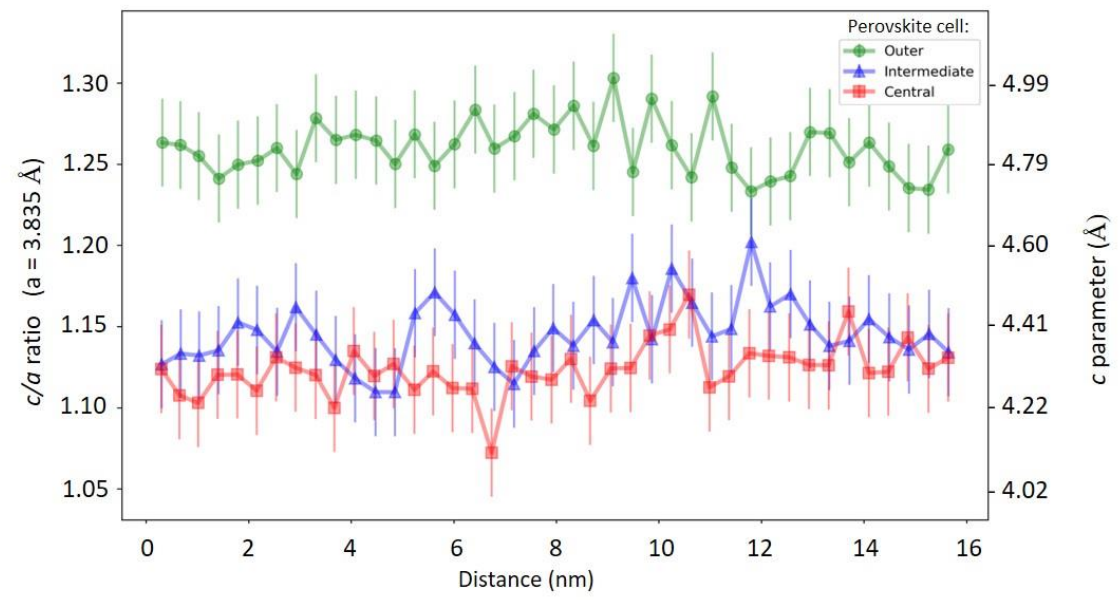

Figure 2: (a) Polarisation map across a head-to-head charged domain wall (DW). The arrow lengths indicate the relative magnitude of the B-site displacement and hence the relative magnitude of the polarisation. A yellow dashed line indicates the approximate DW position. The polarisation magnitudes are suppressed near the DW, indicating a width of approximately 5-8 PK-cells. (b) Map of perovskite (PK)-cell angles reveals a $\sim 6^{\circ}$ shearing at the DW. (c) map and (d) profiles of c/a ratio comparing the outer, intermediate and central PK-cells. The c/a ratio is measured with respect to the average $a$ parameter of $3.835 \AA$, making $c / a$ equal to strain $\varepsilon_{y y}+1$. Error bars represent estimated measurement uncertainty. The measured $a$ parameter varies $\pm 4 \%$ within the ferroelectric layer. This alters the actual measured $c / a$ values from those on the graph, but introduces more error while at the same time not changing the relationship between outer, intermediate and central PK-cells. See Supplementary Figure 3 for complete results. Scalebars $=2 \mathrm{~nm}$. 
Figure 2 displays the detail of the PK-cell polarisation evolution across the $180^{\circ} \mathrm{H}-\mathrm{H} \mathrm{DW}$. The lowest energy polarisation state is to point to one of the "corner" Bi atoms of the PK-cell[29]. This appears to contribute to the angled orientation of the domain wall, seen throughout the figures in this study. The yellow traced domain wall position is approximated as running between the inflection point of the polarisation in the outer PK-cells. The 5-8 PK-cells of inhomogeneous net polarisation is similar in width to charged $\mathrm{DWs}$ in $\mathrm{BiFeO}_{3}$ but quite large for a FE domain wall[34]. This infers a lower exchange energy allowed by a lower anisotropy cost. That is to say, the central PK-cells seem to be more "polarisable" than structurally harder FE's with thinner DWs such as $\mathrm{PbTiO}_{3}$ [35]. The anisotropy cost of the DW is studied by examining rotation and $\varepsilon_{y y}$ strain maps in Figure 2(b) and Figure 2(c), respectively. While no clear change in $\varepsilon_{y y}$ strain was identified at the DW in Figure 2(c), the strong rotation of $\sim 6^{\circ}$ in Figure 2 (b) indicates a shearing response of the structure at the DW. The same effect was seen in $\mathrm{BiFeO}_{3} \mathrm{H}-\mathrm{T}$ DWs[34]. The lack of strain difference between domains confirms that there is little anisotropy cost, allowing for a semi-continuous rotation of the polarisation i.e. Néel/Bloch-type behaviour [36]. The property of central layers being "polarisable" is associated with negative capacitance, specifically in FE-DE multilayer heterostructures[37]. However, in this case, the PK-cell retains a larger $c / a$ ratio than pseudo-cubic bulk rhombohedral BFO so the effect is deemed unlikely. The demonstrated "structural softness" is consistent with a large magnetoelectric effect[38]. Furthermore, the relatively low anisotropy associated with the domain wall indicates that switching is determined only by the electrostatic energy, with no elastic energy cost. These results imply that charged DWs in B6TFMO are likely to be highly mobile with low dielectric loss.

While in bulk BFO, $c=a=3.96 \AA$, epitaxial strain $\left(\varepsilon_{\mathrm{xx}}\right)$ can result in rhombohedral and tetragonal phases with $c / a$ ratios up to 1.13 and 1.27 respectively [39]. Fascinatingly, the measured $a$ spacing in B6TFMO (range: $3.68 \AA$ to $3.99 \AA$, uncertainty +/- $0.1 \AA$, average: 3.835 $\AA$,) includes the $a$ spacing of $\mathrm{LaAlO}_{3}$ at $3.79 \AA$ on which the rhombohedral $(a>3.79 \AA$ ) and tetragonal ( $a<3.79 \AA$ A $)$ BFO phases can co-exist $[39,40]$. This implies that individual PK-cells in B6TFMO can exist flexibly in local rhombohedral or tetragonal phases. The natural $a$ spacing of the $\left(\mathrm{Bi}_{2} \mathrm{O}_{2}\right)^{2+}$ DE layer is calculated to be $3.80 \AA$. The average $a$ value agrees exactly with previous AP measurements, falling between the natural DE layer and PK-cell $a$ values [41]. Therefore the outer PK-cells bonded to the DE layer are effectively under compressive 
epitaxial stress/strain $\left(-\varepsilon_{\mathrm{xx}}\right)$ for their a parameter. Thus, in Figure 2(c), the observation of $c / a$ $\approx 1.27$ in the outer PK-cells can be explained as a transition to the hyper-tetragonal phase of BFO/B6TFMO's PK-cell. Similarly, the observed $c / a \approx 1.10-1.15$ in the central and intermediate PK-cells corresponds to a highly strained $\left(+\varepsilon_{y y},-\varepsilon_{x x}\right)$ rhombohedral BFO/B6TFMO's PK-cell. Therefore, a unit cell of B6TFMO can be considered as containing a morphotropic phase boundary between rhombohedral and tetragonal PK phases. Keeney et al found that the B-site cations separated either side of the boundary in B6TFMO, with magnetic Mn preferring to occupy the central and intermediate PK-cells and Ti preferring to occupy the outer PK-cells[19].

(a)

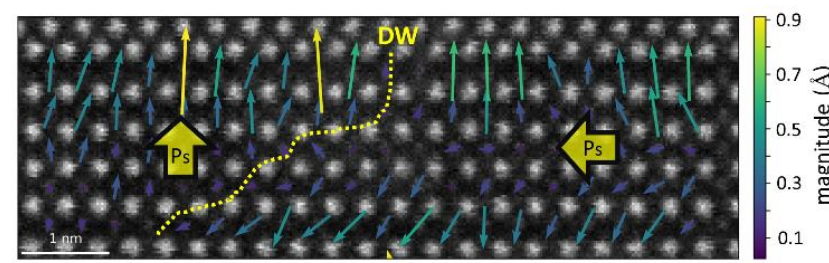

(b)

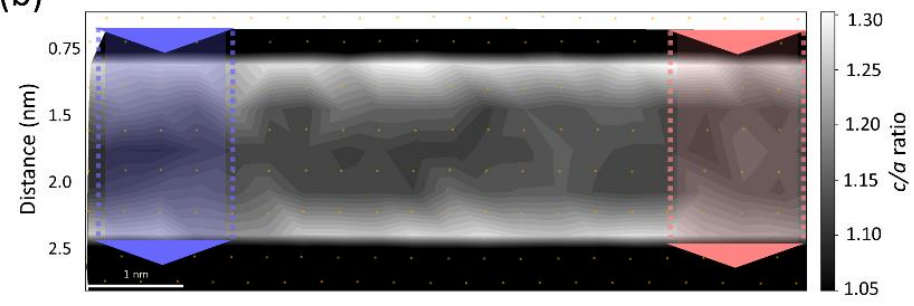

(c)

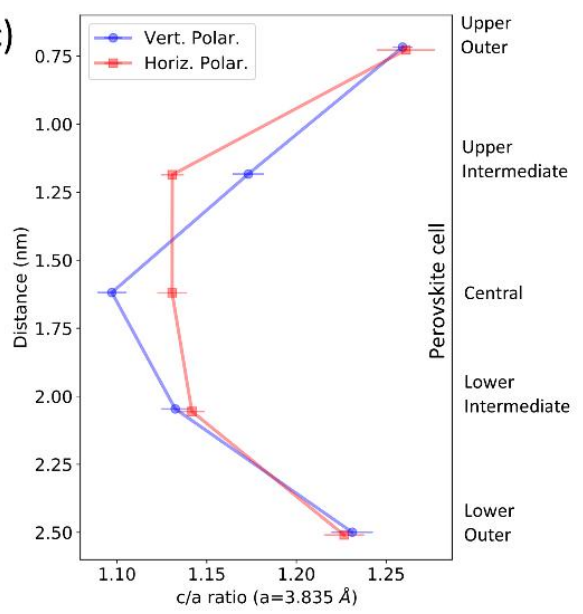

Figure 3: (a) Polarisation map of a head-to-tail domain wall. Vectors are coloured by magnitude of the B-site displacement. Polarisation magnitudes are suppressed in the lower perovskite (PK)-cells and enhanced in the upper PK-cells in the left domain. This results in a net vertical, c-axis polarisation as indicated by the large yellow arrow in the left domain. (b) c/a ratio map of (a) and (c) graph comparing line profiles across the c-axis polarised "vertical" domain (blue) and the in-plane polarized "horizontal" domain (red) in (b). The shaded area between dotted lines marks the position of the profiles. The strain values in (c) are averaged over 5 PK-cells, indicated by the arrow widths in (b). In (c) there is a clear asymmetry in the vertically polarized domain, demonstrating the link between strain and polarisation in the structure. Error bars represent standard error of the mean. Scalebars $=1 \mathrm{~nm}$.

In Figure 3(a) a second type of DW is analysed, where the net polarisation is head-to-tail $(\mathrm{H}$ T). There is a stark difference in configuration compared to the $\mathrm{H}-\mathrm{H}$ DWs. Firstly, the net 
polarisation transition across the DW is sharp. Secondly, polarisation magnitudes are suppressed in the lower PK-cells and enhanced in the upper PK-cells in the left domain. This results in a net vertical, c-axis polarisation. $\mathrm{H}-\mathrm{T}$ DWs are the most commonly observed type of DW in normal ferroelectrics, but the degree of out-of-plane polarisation is unexpected. In odd-layered AP materials, perfect mirroring along the c-axis is not possible due to symmetry and octahedral rotations. The sole contributor to out-of-plane polarisation is therefore expected to be the symmetry breaking central PK-cell, with mirrored polarisation of the outer and intermediate PK-cells[18,42-44]. What we observe in the left domain of Figure $3(a)$ is completely different; polarisation is almost uniformly pointing up in every PK-cell. The lower PK-cells have a suppressed polarisation magnitude (B-site displacement) $\sim 0.1 \AA$, while the upper PK-cells have an enhanced polarisation magnitude of $\sim 0.7 \AA$. For reference, the polarisation magnitude in bulk BFO is $\sim 0.2 \AA$. Faraz et al measured an out-of-plane FE response of $10 \mathrm{pm} / \mathrm{V}$ in these films[15]. Furthermore, there is correlation between this asymmetric c-axis polarisation and the $\varepsilon_{y y}$ strain of the FE layer in Figure 3(c). For the vertically polarised domain in Figure 3(a), the larger polarisation vectors for the upper-outer and upperintermediate PK-cells correspond to increased $\varepsilon_{y y}$ strain in Figure 3(c), compared with the inplane polarised domain. While there is less $\varepsilon_{y y}$ strain in the central PK-cell compared with the in-plane domain, there is no $\varepsilon_{y y}$ strain difference between vertical and in-plane domains for the intermediate and outer PK-cells at the bottom of the layer, where the polarisation is suppressed. For the horizontally polarised domain in Figure 3 , both $\varepsilon_{y y}$ strain and polarisation vectors are symmetrical. The difference in strain states between the two domain types means there is a relatively high anisotropy cost for the DW. This manifests itself in the sharp, welldefined, $\mathrm{H}-\mathrm{T}$ transition across 1-2 PK-cells, contrasting with the wider $\mathrm{H}-\mathrm{H}$ DW in Figure 2, but still following the same angled orientation. From EELS mapping (Supplementary Figure 4) there is a clear indication of oxygen depletion at the DW. Oxygen vacancies are common in perovskites, and normally found even at H-T DWs purely due to the strain field present, without needing to provide electrostatic screening $[45,46]$. 

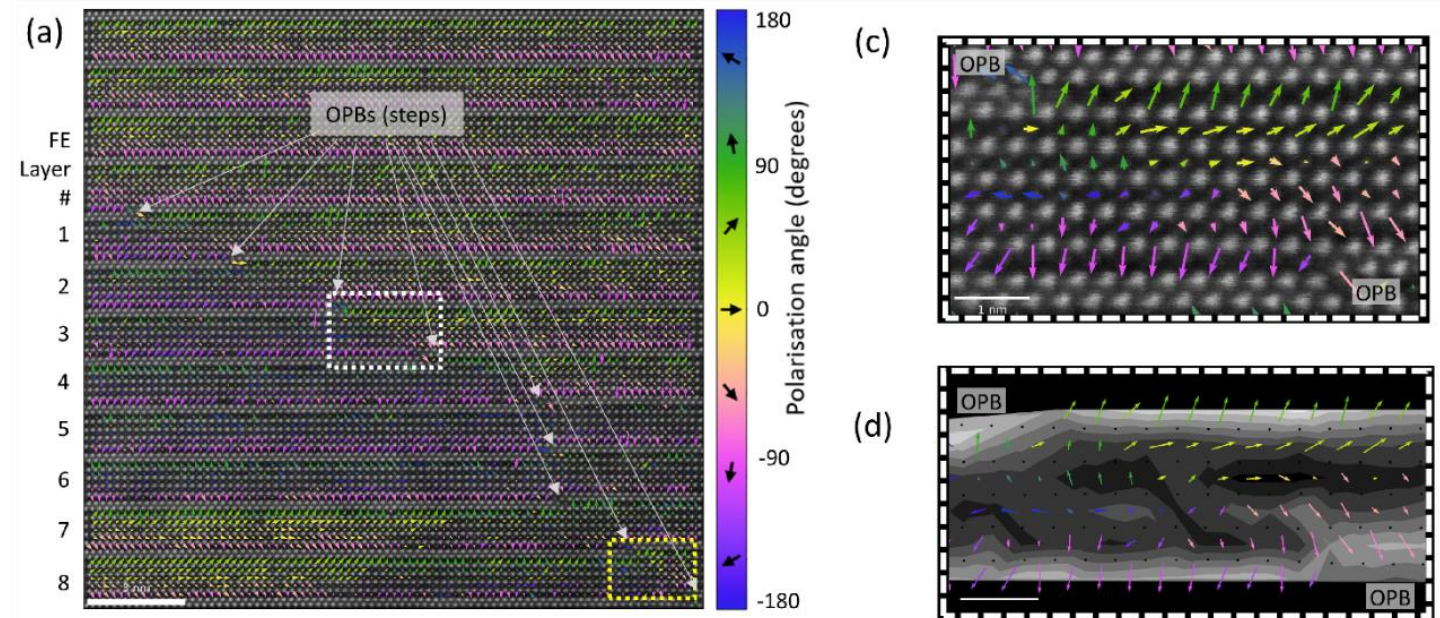

(d)

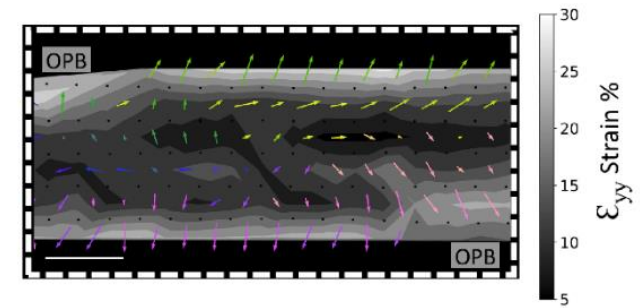

(b)

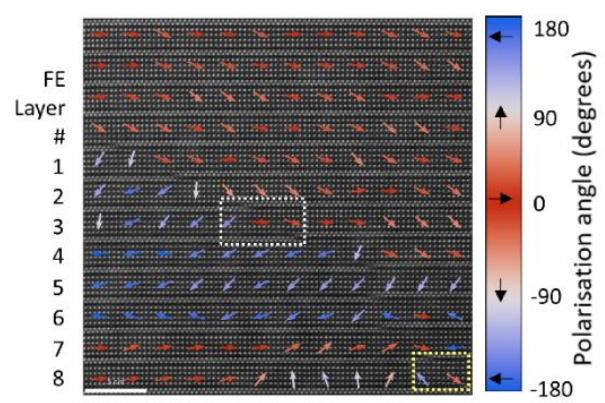

(e)

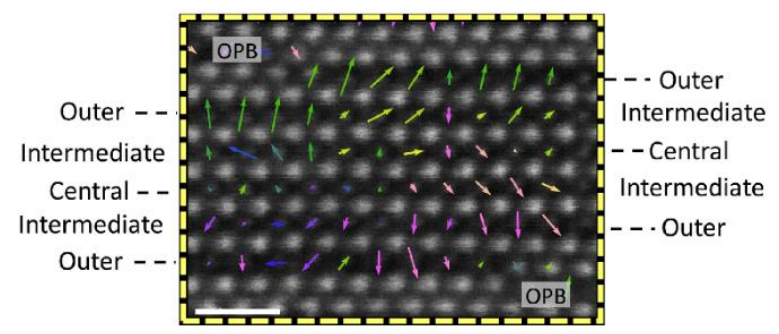

Figure 4 (a) Polarisation map of a large area containing domain walls (DWs) pinned at out-of-phase boundaries (OPBs). The polarisation vector colours represent their angle relative to the $x$-axis. Two vortexes are present, marked by dashed boxes. (b) The same map as (a), with polarisation vectors summed over $5 \times 5$ perovskite (PK)-cells and colour coded red/blue to demonstrate the tail-to-tail (T-T) character of the DWs. (c) Enlarged area from the white dashed box in (a) detailing the polarisation vortex which forms where T-T DWs occur in the vicinity of OPBs. (d) The polarisation vectors from (b) overlaid on a $\varepsilon_{y y}$ strain map of the same area, with black dots representing the Bi atomic positions. $\varepsilon_{y y}$ strain is defined at the change of the $c$ parameter from the average a parameter $(3.835 \AA$ ) , with $0 \%$ representing a pseudo-cubic structure. (e) Enlarged area from the yellow dashed box in (a) where another vortex has formed. PK-cells are labelled to demonstrate the change in structure at the OPB. (c) and (e) share the same polarisation colour bar as (a). Scalebars in (a, b) $=5 \mathrm{~nm}$, scalebars in (c, d, e) $=1 \mathrm{~nm}$. See Supplementary Figures 5-7 for more details.

A further illustration of the interplay between strain and polarisation is shown in Figure 4, where a FE polarisation vortex is identified. The feature can be seen in atomic level detail in Figure $4(\mathrm{c})$. The vortex occurs as a form of $180^{\circ}$ polarisation transition between tail-to-tail (TT domains). As such, the vortex must be charged to the same extent as the $\mathrm{H}-\mathrm{H}$ DW in Figure 2 , with average $\sigma_{\mathbf{s}}=1.09$ e per PK-cell. Although the DW width is similar to the $\mathrm{H}-\mathrm{H}$ case in Figure 2, the defined vortex polarisation suggests the charge density is concentrated at the 
vortex core [47], further increasing the relative conductivity difference versus the domain. The required positive charge may be provided by either oxygen vacancies or a local change in oxidation state:

$\mathrm{Ti}^{4+}->\mathrm{Ti}^{3+}+1 h^{+}$or $[\mathrm{Bi} / \mathrm{Mn} / \mathrm{Fe}]^{3+}->[\mathrm{Bi} / \mathrm{Mn} / \mathrm{Fe}]^{2+}+1 h^{+}$, where $h^{+}$represents an electron hole.

Two of these vortexes occur in a region of the B6TFMO film containing "out-of-phase boundary" (OPB) defects, as shown in Figure 4(a,b). OPBs are common boundary defects occurring in materials of high structural anisotropy, such as the Aurivillius phases, and are characterized by displacement by a fraction of a lattice parameter $(c / x)$ between two neighbouring regions parallel to the c-direction [48].. They appear as a "step" in the DE and FE layer and are energetically preferred to dislocations for relieving out-of-plane stress in the film. Disruptions of the AP lattice by OPBs have a marked influence on the elastic strain energies within the structure which may drive larger cations away from the OPB [19]. From Figure $4(a)$ it is evident that the line of OPBs propagates diagonally down through the film, similar to the preferred orientation of the $\mathrm{H}-\mathrm{H}$ DW in Figure 2. Figure 4(b), in which the polarisation vectors are summed over $5 \times 5$ PK-cells, shows that the T-T DW broadly follows the OPBs down through the layers of the film. The strain gradient around the OPB is likely a preferential site for vacancies, providing some screening charge to help stabilise the charged DW. The T-T DW decouples from the OPBs in FE-layers 5-7, which instead contain a $180^{\circ}$ neutral DW and a $180^{\circ} \mathrm{H}-\mathrm{H}$ DW. FE layer 8 contains both a H-H DW and a T-T vortex. This is shown in detail in Figure $4(\mathrm{~d})$. The rich variety of features within just $20 \mathrm{~nm}$ is testament to the complex electrostatic screening in the film.

Vortexes and vortices are more traditionally associated with ferromagnetic domains, where there is no anisotropy cost to extend the transition of the magnetisation direction, thus lowering the exchange energy at the DW[49]. FE vortexes/vortices have previously been identified in thin films of $\mathrm{BFO}$ and $\mathrm{PbTiO}_{3}$ multilayers [50-55] but remain a novel occurrence. To examine the strain state of the vortex we compare the c-axis strain map ( $\left.\varepsilon_{y y}\right)$ in Figure $4(d)$ to the PK-cell polarisation in Figure 4(c). There is an asymmetric $\varepsilon_{y y}$ strain gradient either side of the vortex, but no strain change at its centre. Thus the anisotropy cost, DW width and the exchange energy (determined by the curl of the polarisation) should show the same 
behaviour as the $\mathrm{H}-\mathrm{H}$ DW in Figure 2 . It appears that the vortex occurs due to geometric/strain effects controlling the polarisation as demonstrated in Figure 4(d). Increased $\varepsilon_{y y}$ strain to the upper-left and lower-right of the vortex core corresponds to OPBs adding an extra PK-cell polarised up and down respectively on either side, i.e. the increased $\varepsilon_{y y}$ strain is caused by the "steps". Furthermore, the central PK-cells which provide the in-plane polarisation are displaced. In Figure 4(c \& e), when the lower central PK-cell on the left becomes the intermediate PK-cell after the OPB, the polarisation turns downward. When the upper central PK-cell on the right becomes the intermediate PK-cell after the OPB, the polarisation turns upward. Thus, the $180^{\circ} \mathrm{T}-\mathrm{T} \mathrm{DW}$, instead of behaving similar to the H-H DW in Figure 2, becomes a vortex with a well-defined curl of the polarisation. In line with this explanation, we observe that the lateral distance between successive OPBs must be similar to the $180^{\circ}$ charged DW width, 5-8 PK-cells, for the vortex to form. The factors affecting vortex formation in each of the FE-layers \#1-8 in Figure 4(a) are detailed in Table 1.

\begin{tabular}{|l|l|l|l|}
\hline FE Layer & $\begin{array}{l}\text { Charged DW present } \\
\text { between OPBs? }\end{array}$ & $\begin{array}{l}\text { OPBs spaced 5-8 PK- } \\
\text { cells apart? }\end{array}$ & Vortex present? \\
\hline 1 & Yes & No & No \\
\hline 2 & Yes & No & No \\
\hline 3 & Yes & Yes & Yes \\
\hline 4 & Yes & No & No \\
\hline 5 & No & No & No \\
\hline 6 & No & No & No \\
\hline 7 & No & Yes & No \\
\hline 8 & Yes & Yes & Yes \\
\hline
\end{tabular}

Table 1: Comparison of the factors affecting vortex formation in the FE layers containing OPBs presented in Figure 4(a,b).

These results offer a detailed insight into the ferroelectric behaviour of B6TFMO and AP materials. However, at the nanoscale, there remains significant questions to be answered. For instance, the structure of AP materials offers intriguing hints pointing towards localised charge separation. Each FE layer has a sharp transition from up to down polarisation across the 5 PK-cells and thus could be considered to contain a horizontal DW in the FE layer. The central PK-cell should then contain positive screening charge, with the DE layer containing 
negative screening charge. Interestingly, Keeney et al identified that $\mathrm{Mn}$ has a preference for concentrating towards the central PK-cell and thus plays a key role generating room temperature ferromagnetism [19]. $\mathrm{Mn}^{3+}$, the nominal oxidation state in B6TFMO to achieve charge balance, is known to disproportionate into $\mathrm{Mn}^{2+}$ and $\mathrm{Mn}^{4+}$ [56]. The transition to $\mathrm{Mn}^{2+}$ and $\mathrm{Mn}^{4+}$ provides a likely source of electrons and holes for charge screening. Charge separation between the centre and edges of each FE layer is thus a potentially interesting phenomenon to be further investigated.

Moving from the PK-cell to the FE layer scale, there is evidence of polarisation coupling through multiple FE layers in each of the multilayer maps. The DE layers do not fully isolate the FE layers. One should consider whether FE behaviour may be altered in a cross-section lamella ( $<35 \mathrm{~nm}$ in thickness to enable HR-STEM analysis) compared to the as-grown thin film. Thin lamellae strongly favour polarisation within the plane of the lamella to reduce surface charging. In the film, the polarisation can rotate freely towards the viewing axis, adding an extra degree of freedom to the system. PFM results from other AP materials show that charged domain walls are evident in as-grown films[3], so the FE results from STEM HAADF presented here appear to be representative. The conduction mechanisms in similar AP films vary depending on the strength of the applied field but both ionic conductivity and electron hopping conductivity have an important contribution according to Song et al[57].

Finally we consider the presence of ferromagnetism in the structure and how it would react to the FE DWs identified. Magnetoelectric coupling should be mediated under the Dzyaloshinskii-Moriya (DM) interaction[58]. In line with GMR film behaviour, that would suggest the magnetisation preferentially orienting in-plane in the FE layer but perpendicular to the FE polarisation. The coupling strength remains to be investigated, and depends on whether the DM interaction is stronger than the exchange energy penalty for matching the curl of the magnetisation to the curl of the polarisation across the DWs. It is likely that the exchange energy dominates, resulting in a wider magnetic DW mirroring the FE DW[59]. The magnetisation could even be locally enhanced if it does not invert with the FE polarisation [60]. Answering these questions is extremely important in realising electric field control of magnetisation and magnetoresistance. Quantifying the relationship will be the subject of future work. 


\section{Conclusion}

Aurivillius phase B6TFMO contains a naturally occurring dielectric-ferroelectric heterostructure and a morphotropic phase boundary, meaning distinctive ferroelectric behaviour is intrinsic. Both $\mathrm{H}-\mathrm{H}$ and T-T domain walls are present below the surface of B6TFMO thin films and are coupled between ferroelectric layers and are not altogether electrostatically isolated by the DE layers in between. They are highly charged with average $\sigma_{\mathbf{s}}=1.09|\mathrm{e}|$ per perovskite-cell and $\rho \sim 0.17|\mathrm{e}|$ per perovskite-cell. The exact nature of screening charge is yet to be determined but local charge separation is likely due to the intrinsically opposing polarisation within the ferroelectric layer. H-T domains exhibit c-axis polarisation and the accompanying strain asymmetry signals electromechanical coupling. Out-of-phase boundary defects were observed to induce complete ferroelectric polar vortexes, if separated by a distance similar to the DW width.

The vortexes give insight into the scale and magnitude at which locally induced strain can influence polarisation rotation. The observations relating to anisotropy and exchange energy at different domain walls can inform future investigations into the magnetoelectric coupling and ferromagnetic domain characteristics in B6TFMO. These polar topological solitons form only at the OPBs. This suggests there is a link between the increased magnetoelectric effects measured by others in the AP community and the formation of polar vortexes and charged domain walls we present in this paper. These results highlight the Aurivillius phase as an ideal platform for designing useful DW-based multiferroic materials.

\section{Methods}

\section{Crystal Growth:}

B6TFMO on c-sapphire substrates by liquid injection chemical vapour deposition (LI-CVD) methods[61] and post-annealed at $850^{\circ} \mathrm{C}(1123 \mathrm{~K})$. The average stoichiometry, as determined by HR-SEM (high resolution scanning electron microscopy) with EDX, was $\mathrm{Bi}_{6} \mathrm{Ti}_{2.8} \mathrm{Fe}_{1.52} \mathrm{Mn}_{0.68} \mathrm{O}_{18}$. 


\section{Electron Microscopy:}

Cross-sections of the B6TFMO films were prepared using a FEI Dual Beam Helios NanoLab 600i focused ion beam (FIB) and were mounted on a Cu based TEM grid. The sample was thinned via the FIB Ga beam first at $30 \mathrm{kV}$ and $93 \mathrm{pA}$, and then $5 \mathrm{kV}$ and $43 \mathrm{pA}$. The samples were then further thinned and polished to sub> 30nm using a Fischione $1020 \mathrm{Ar}$ ion based plasma cleaner prior to STEM imaging and EDX/EELS analysis. Energy filtered images acquired at $300 \mathrm{kV}$ on an FEl Titan TEM with Gatan Tridiem Energy Filtering system demonstrated that thicknesses of the regions used for imaging were $<30 \mathrm{~nm}$. Imaging and analysis was performed on a NION UltraSTEM 200 operating at 200 kV. A Gatan Enfinium and GMS 2.0 was used for EELS acquisition and analysis.

Atom position finding and 2D Gaussian refinement were completed with the Atomap Python package[62]. Image analysis and mapping, as well as polarisation vector analysis, were completed using the TEMUL Toolkit Python package[63]. Supplementary material strain analysis was carried out by geometric phase analysis using the Stem Cell program [64].

\section{Acknowledgements:}

This research was supported by the US-Ireland R\&D Partnership Programme (grant no. USI $120)$ and the Science Foundation Ireland (16/US/3344). M.C. acknowledges funding from SFI Industry Fellowship (18/IF/6282). L. K. acknowledges funding from the Royal Society and Science Foundation Ireland (SFI) University Research Fellowship UF 140263.

\section{Corresponding Authors:}

*Correspondence should be addressed to K. M. (kalanimoore@gmail.com) and M. C. (michele.conroy@ul.ie)

\section{Ethics Declaration:}

The authors declare no competing financial or non-financial interests. 


\section{References:}

[1] Keeney, L., Schmidt, M., Amann, A., Maity, T., Deepak, N., Faraz, A., Petkov, N., Roy S., Pemble, M. E., Whatmore, R. W., 2016 Novel Approaches for Genuine Single-Phase Room Temperature Magnetoelectric Multiferroics Nanoscale Ferroelectr. Multiferroics 789-829

[2] Hervoches, C. H., Snedden, A., Riggs, R., Kilcoyne, S.H., Manuel. P., Lightfoot, P., 2002 Structural behavior of the four-layer aurivillius-phase ferroelectrics SrBi4Ti4O15 and Bi5Ti3FeO15 J. Solid State Chem. 164 280-91

[3] Gradauskaite, E., Campanini, M., Biswas, B., Schneider, C. W., Fiebig, M., Rossell, M. D., Trassin, M., 2020 Robust In-Plane Ferroelectricity in Ultrathin Epitaxial Aurivillius Films Adv. Mater. Interfaces 2000202

[4] De Araujo, C. A. P., Cuchiaro, J. D., Mc Millan, D. L., Scott, M. C., Scott, J. F., 1995 Fatigue-free ferroelectric capacitors with platinum electrodes Nature 374 627-9

[5] Park, B., Kang, B., Bu, S., Noh, T., Lee, J., Jo, W., 1999 Lanthanum-substituted bismuth titanate for use in non-volatile memories Nature

[6] Pan, Z., Wang, P., Hou, X., Yao, L., Zhang, G., Wang, J., Liu, J., Shen, M., Zhang, Y., Jiang, S., Zhai, J., Wang, Q., 2020 Fatigue-Free Aurivillius Phase Ferroelectric Thin Films with Ultrahigh Energy Storage Performance Adv. Energy Mater. 10

[7] Yang, B. B., Song, D. P., Wei, R. H., Tang, X. W., Hu, L., Yang, J., Song, W. H., Dai, J. M., Zhu, X. B., Sun, Y. P., $2018 \mathrm{Ni}$ doping dependent dielectric, leakage, ferroelectric and magnetic properties in Bi 7 Fe 3-x Ni x Ti 3021 thin films Appl. Surf. Sci. 440 484-90

[8] Zhai, X., Grutter, A. J., Yun, Y., Cui, Z., Lu, Y., 2018 Weak magnetism of Aurivillius-type multiferroic thin films probed by polarized neutron reflectivity Phys. Rev. Mater. 2 044405

[9] Birenbaum, A. Y., Ederer, C., 2014 Potentially multiferroic Aurivillius phase $\mathrm{Bi}_{5} \mathrm{FeTi}_{3}$ $\mathrm{O}_{15}$ : Cation site preference, electric polarization, and magnetic coupling from first principles Phys. Rev. B - Condens. Matter Mater. Phys. 90214109

[10] Algueró, M,. Pérez-Cerdán, M., del Real, R. P., Ricote, J., Castro, A., 2020 Novel Aurivillius $\mathrm{Bi}_{4} \mathrm{Ti}_{3-2 \times} \mathrm{Nb}_{x} \mathrm{Fe}_{\times} \mathrm{O}_{12}$ phases with increasing magnetic-cation fraction until percolation: a novel approach for room temperature multiferroism J. Mater. Chem. $C$ 8 12457-69

[11] Koval, V., Skorvanek, I., Viola, G., Zhang, M., Jia, C ., Yan, H., 2018 Crystal Chemistry and Magnetic Properties of Gd-Substituted Aurivillius-Type $\mathrm{Bi}_{5} \mathrm{FeTi}_{3} \mathrm{O}_{15}$ Ceramics J. Phys. Chem. C 122 15733-43

[12] Manipatruni, S, Nikonov, D., Young, I., 2020 RECURRENT NEURON IMPLEMENTATION BASED ON MAGNETO-ELECTRIC SPIN ORBIT LOGIC

[13] Manipatruni, S., Nikonov, D. E., Lin, C. C., Gosavi, T. A., Liu, H., Prasad, B., Huang, Y. L, Bonturim, E., Ramesh, R., Young, I. A., 2019 Scalable energy-efficient magnetoelectric spin-orbit logic Nature 565 35-42 
[14] Keeney, L., Maity, T., Schmidt, M., Amann, A., Deepak, N., Petkov, N., Roy, S., Pemble, M. E., Whatmore, R. W., 2013 Magnetic field-induced ferroelectric switching in multiferroic aurivillius phase thin films at room temperature J. Am. Ceram. Soc. 96 2339-57

[15] Faraz, A., Maity, T., Schmidt, M., Deepak, N., Roy, S., Pemble, M. E., Whatmore, R. W., Keeney, L., 2017 Direct visualization of magnetic-field-induced magnetoelectric switching in multiferroic aurivillius phase thin films J. Am. Ceram. Soc. 100 975-87

[16] Gross, I., Akhtar, W., Garcia, V., Martínez, L. J., Chouaieb, S., Garcia, K., Carrétéro, C., Barthélémy, A., Appel, P., Maletinsky, P., Kim, J. V., Chauleau, J. Y., Jaouen, N., Viret, M., Bibes, M., Fusil, S., Jacques, V., 2017 Real-space imaging of non-collinear antiferromagnetic order with a single-spin magnetometer Nature 549 252-6

[17] Hervoches, C. H., Lightfoot, P., 1999 A variable-temperature powder neutron diffraction study of ferroelectric $\mathrm{Bi}_{4} \mathrm{Ti}_{3} \mathrm{O}_{12}$ Chem. Mater. 11 3359-64

[18] Shrinagar, A., Garg, A., Prasad, R., Auluck, S., 2008 Phase stability in ferroelectric bismuth titanate: A first-principles study Acta Crystallogr. Sect. A Found. Crystallogr. $64368-75$

[19] Keeney, L., Downing, C., Schmidt, M., Pemble, M. E., Nicolosi, V., Whatmore, R. W., Direct atomic scale determination of magnetic ion partition in a room temperature multiferroic material The five-layer Aurivillius phase Bi 6 Ti x Fe y Mn z OPEN

[20] Pereira Gonçalves, M. A., Escorihuela-Sayalero, C., Garca-Fernández, P., Junquera, J., Íniguez, J., 2019 Theoretical guidelines to create and tune electric skyrmion bubbles Sci. Adv. 5 eaau 7023

[21] Mandal, P., Pitcher, M. J., Alaria, J., Niu, H., Borisov, P., Stamenov, P., Claridge, J. B., Rosseinsky, M. J., 2015 Designing switchable polarization and magnetization at room temperature in an oxide Nature 525 363-6

[22] Mundy, J. A., Schaab, J., Kumagai, Y., Cano, A., Stengel, M., Krug, I. P., Gottlob, D. M., Doğanay, H., Holtz, M. E., Held, R., Yan, Z., Bourret, E., Schneider, C. M., Schlom, D. G., Muller, D. A., Ramesh, R., Spaldin, N. A., Meier, D., 2017 Functional electronic inversion layers at ferroelectric domain walls Nat. Mater. 16 622-7

[23] Mundy, J. A., Heikes, C. A., Grosso, B. F., Segedin, D. F., Wang, Z., Goodge, B. H., Meier, Q. N., Nelson, C. T., Prasad, B., Kourkoutis, L. F., Ratcliff, W. D., Spaldin, N. A., Ramesh, R., Schlom, D. G., 2018 A high-energy density antiferroelectric made by interfacial electrostatic engineering

[24] Kumar, A., Baker, J. N., Bowes, P. C., Cabral, M. J., Zhang, S., Dickey, E. C., Irving, D. L., LeBeau, J. M., 2020 Atomic-resolution electron microscopy of nanoscale local structure in lead-based relaxor ferroelectrics Nat. Mater. 1-6

[25] Jia, C. L., Mi, S. B., Urban, K., Vrejoiu, I., Alexe, M., Hesse, D., 2008 Atomic-scale study of electric dipoles near charged and uncharged domain walls in ferroelectric films Nat. Mater. 7 57-61

[26] El Baggari, I., Savitzky, B. H., Admasu, A. S., Kim, J., Cheong, S. W., Hovden, R., Kourkoutis, L. F., 2018 Nature and evolution of incommensurate charge order in 
manganites visualized with cryogenic scanning transmission electron microscopy Proc. Natl. Acad. Sci. U. S. A. 115 1445-50

[27] Daumont, C., Ren, W., Infante, I. C., Lisenkov, S., Allibe, J., Carrétéro, C., Fusil, S., Jacquet, E., Bouvet, T., Bouamrane, F., Prosandeev, S., Geneste, G., Dkhil, B., Bellaiche, L., Barthélémy, A., Bibes, M., 2012 Strain dependence of polarization and piezoelectric response in epitaxial BiFeO 3 thin films J. Phys. Condens. Matter 24 162202

[28] Schilling, A., Bowman, R. M., Catalan, G., Scott, J. F., Gregg, J. M., 2007 Morphological Control of Polar Orientation in Single-Crystal Ferroelectric Nanowires Nano Lett 7 3787-91

[29] Campanini, M., Trassin, M., Ederer, C., Erni, R., Rossell, M. D., 2019 Buried In-Plane Ferroelectric Domains in Fe-Doped Single-Crystalline Aurivillius Thin Films ACS Appl. Electron. Mater. 1 1019-28

[30] Bednyakov, P. S., Sturman, B. I., Sluka, T., Tagantsev, A. K., Yudin, P. V., 2018 Physics and applications of charged domain walls npj Comput. Mater. 465

[31] Liu, Y., Zhu, Y. L., Tang, Y. L., Wang, Y. J., Li, S., Zhang, S. R., Han, M. J., Ma, J. Y., Suriyaprakash, J., Ma, X. L., 2017 Controlled Growth and Atomic-Scale Mapping of Charged Heterointerfaces in $\mathrm{PbTiO} 3 / \mathrm{BiFeO} 3$ Bilayers ACS Appl. Mater. Interfaces 9 25578-86

[32] Scott, J. F., 2007 Multiferroic memories Nat. Mater. 6 256-7

[33] Yin, Y. W., Raju, M., Hu, W. J., Weng, X. J., Zou, K., Zhu, J., Li, X. G., Zhang, Z. D., Li, Q., 2012 Multiferroic tunnel junctions Front. Phys. 7 380-5

[34] Rojac, T., Bencan, A., Drazic, G., Sakamoto, N., Ursic, H., Jancar, B., Tavcar, G., Makarovic, M., Walker, J., Malic, B., Damjanovic, D., 2017 Domain-wall conduction in ferroelectric BiFeO 3 controlled by accumulation of charged defects

[35] Catalan, G., Seidel, J., Ramesh, R., Scott, J. F., 2012 Domain wall nanoelectronics Rev. Mod. Phys. 84 119-56

[36] Bauer, A., Pfleiderer, C., 2016 Topological Structures in Ferroic Materials "Topological Struct. Ferroic Mater. Springer Ser. Mater. Sci. 228 1-28

[37] Zubko, P., Wojdeł, J. C., Hadjimichael, M., Fernandez-Pena, S., Sené, A., Luk'yanchuk, I., Triscone, J. M. Íñiguez, J., 2016 Negative capacitance in multidomain ferroelectric superlattices

[38] Wojdeł, J. C., Íñiguez, J., 2010 Ab initio indications for giant magnetoelectric effects driven by structural softness Phys. Rev. Lett. 105037208

[39] Huang, R., Ding, H. C., Liang, W. I., Gao, Y. C., Tang, X. D., He, Q., Duan, C. G., Zhu, Z., Chu, J., Fisher, C. A. J., Hirayama, T., Ikuhara, Y., Chu, Y. H. 2014 Atomic-Scale Visualization of Polarization Pinning and Relaxation at Coherent BiFeO3/LaAlO3 Interfaces Adv. Funct. Mater. 24 793-9

[40] Béa H, Dupé B, Fusil S, Mattana R, Jacquet E, Warot-Fonrose B, Wilhelm F, Rogalev A, 
Petit S, Cros V, Anane A, Petroff F, Bouzehouane K, Geneste G, Dkhil B, Lisenkov S, Ponomareva I, Bellaiche $L$, Bibes $M$ and Barthélémy A 2009 Evidence for roomtemperature multiferroicity in a compound with a giant axial ratio Phys. Rev. Lett. 102 217603

[41] Armstrong, R. A., Newnham, R. E., 1972 Bismuth titanate solid solutions Mater. Res. Bull. 7 1025-34

[42] Moure, A., 2018 Review and Perspectives of Aurivillius Structures as a Lead-Free Piezoelectric System Appl. Sci. 862

[43] Pignolet A, Schäfer C, Satyalakshmi K M, Harnagea C, Hesse D and Gösele U 2000 Orientation dependence of ferroelectricity in pulsed-laser-deposited epitaxial bismuth-layered perovskite thin films Appl. Phys. A Mater. Sci. Process. 70 283-91

[44] Newnham R E, Wolfe R W and Dorrian J F 1971 Structural basis of ferroelectricity in the bismuth titanate family Mater. Res. Bull. 6 1029-39

[45] Aschauer U, Pfenninger R, Selbach S M, Grande T and Spaldin N A 2013 Straincontrolled oxygen vacancy formation and ordering in $\mathrm{CaMnO}_{3}$ Phys. Rev. B - Condens. Matter Mater. Phys. 88054111

[46] Farokhipoor S and Noheda B 2012 Local conductivity and the role of vacancies around twin walls of (001)-BiFeO 3 thin films Journal of Applied Physics vol 112 (American Institute of PhysicsAIP) p 052003

[47] Du K, Zhang M, Dai C, Zhou Z N, Xie Y W, Ren Z H, Tian H, Chen L Q, Van Tendeloo G and Zhang $Z 2019$ Manipulating topological transformations of polar structures through real-time observation of the dynamic polarization evolution Nat. Commun. $101-8$

[48] Zurbuchen M A, Tian W, Pan X Q, Fong D, Streiffer S K, Hawley M E, Lettieri J, Jia Y, Asayama G, Fulk S J, Comstock D J, Knapp S, Carim A H and Schlom D G 2007 Morphology, structure, and nucleation of out-of-phase boundaries in epitaxial films of layered oxides J. Mater. Res. 22 1439-71

[49] Catalan G, Seidel J, Ramesh R and Scott J F 2012 Domain wall nanoelectronics Rev. Mod. Phys. 84 119-56

[50] Gregg J M 2012 Exotic Domain States in Ferroelectrics: Searching for Vortices and Skyrmions Ferroelectrics 433 74-87

[51] Yadav A K, Nelson C T, Hsu S L, Hong Z, Clarkson J D, Schlepütz C M, Damodaran A R, Shafer P, Arenholz E, Dedon L R, Chen D, Vishwanath A, Minor A M, Chen L Q, Scott J $F$, Martin L W and Ramesh \& R 2016 Observation of polar vortices in oxide superlattices Nature

[52] Damodaran A R, Clarkson J D, Hong Z, Liu H, Yadav A K, Nelson C T, Hsu S-L, McCarter M R, Park K-D, Kravtsov V, Farhan A, Dong Y, Cai Z, Zhou H, Aguado-Puente P, GarcíaFernández $P$, Íñiguez J, Junquera J, Scholl $A$, Raschke $M B$, Chen L-Q, Fong $D D$, Ramesh R and Martin L W 2017 Phase coexistence and electric-field control of toroidal order in oxide superlattices Nat. Mater. 16 1003-9 
[53] Balke N, Winchester B, Ren W, Chu Y H, Morozovska A N, Eliseev E A, Huijben M, Vasudevan R K, Maksymovych P, Britson J, Jesse S, Kornev I, Ramesh R, Bellaiche L, Chen L Q and Kalinin S V. 2012 Enhanced electric conductivity at ferroelectric vortex cores in BiFeO 3 Nat. Phys. 8 81-8

[54] Nelson C T, Winchester B, Zhang Y, Kim S-J, Melville A, Adamo C, Folkman C M, Baek S-H, Eom C-B, Schlom D G, Chen L-Q and Pan X 2011 Spontaneous Vortex Nanodomain Arrays at Ferroelectric Heterointerfaces Nano Lett 11 828-34

[55] Li Y, Jin Y, Lu X, Yang J C, Chu Y H, Huang F, Zhu J and Cheong S W 2017 Rewritable ferroelectric vortex pairs in $\mathrm{BiFeO}_{3}$ npj Quantum Mater. 243

[56] Hundley M and Neumeier J 1997 Thermoelectric power: Inadequacy of the nominalvalence approach Phys. Rev. B - Condens. Matter Mater. Phys. 55 11511-5

[57] Song D, Yang J, Yang B, Chen L, Wang F and Zhu X 2018 Evolution of structure and ferroelectricity in Aurivillius $\mathrm{Bi}_{4} \mathrm{Bi}_{n-3} \mathrm{Fe}_{n-3} \mathrm{Ti}_{3} \mathrm{O}_{3 n+3}$ thin films J. Mater. Chem. C 6 861827

[58] Park J G, Le M D, Jeong J and Lee S 2014 Structure and spin dynamics of multiferroic $\mathrm{BiFeO}_{3}$ J. Phys. Condens. Matter 26433202

[59] Pyatakov, A. P., Zvezdin, A. K., Vlasov, A. M., Sergeev, A. S., Sechin, D. A., Nikolaeva, E. P., Nikolaev, A. V., Chou, H., Sun, S. J., Calvet, L. E., 2012 Spin structures and domain walls in multiferroics spin structures and magnetic domain walls in multiferroics Ferroelectrics vol 438 pp 79-88

[60] Daraktchiev, M., Catalan, G., Scott, J. F., 2010 Landau theory of domain wall magnetoelectricity Phys. Rev. B - Condens. Matter Mater. Phys. 81224118

[61] Srinivas, A., Suryanarayana, S. V., Kumar, G. S., and Mahesh Kumar M 1999 Magnetoelectric measurements on $\mathrm{Bi}_{5} \mathrm{FeTi}_{3} \mathrm{O}_{15}$ and $\mathrm{Bi}_{6} \mathrm{Fe}_{2} \mathrm{Ti}_{3} \mathrm{O}_{18} \mathrm{~J}$. Phys. Condens. Matter 11 3335-40

[62] Nord, M., Vullum, P. E., MacLaren, I., Tybell, T., Holmestad, R., 2017 Atomap: a new software tool for the automated analysis of atomic resolution images using twodimensional Gaussian fitting Adv. Struct. Chem. Imaging 39

[63] O'Connell E, Hennessy M and Eoin Moynihan 2020 PinkShnack/TEMUL: DOI Release

[64] Grillo V and Rossi F 2013 STEM_CELL: A software tool for electron microscopy. Part 2 analysis of crystalline materials Ultramicroscopy 125 112-29 